\title{
Formation of polarized spectral lines in atmospheres with horizontal inhomogeneities
}

\author{
A. Tichý $\dot{1}^{1,2}$, J. Štěpán ${ }^{1}$, J. Trujillo Bueno ${ }^{3,4,5}$ and J. Kubát ${ }^{1}$ \\ ${ }^{1}$ Astronomical Institute, Academy of Sciences of the Czech Republic, \\ Ondřejov, Czech Republic \\ ${ }^{2}$ Faculty of Science, Masaryk Univerzity, Brno, Czech Republic \\ e-mail: adamt@physics.muni.cz \\ ${ }^{3}$ Instituto de Astrofisica de Canarias, 38205 La Laguna, Tenerife, Spain \\ ${ }^{4}$ Universidad de La Laguna, Departamento de Astrofísica, 38206 La Laguna, Tenerife, Spain \\ ${ }^{5}$ Consejo Superior de Investigaciones Científicas, Spain
}

\begin{abstract}
We study the problem of the generation and transfer of spectral line intensity and polarization in models of stellar atmospheres with horizontal plasma inhomogeneities. We solve the non-LTE radiative transfer problem in full 3D geometry taking into account resonant scattering polarization and its modification by magnetic fields via the Hanle effect. We show that horizontal fluctuations of the thermodynamical conditions of stellar atmospheres can have a significant impact on the linear polarization of the emergent spectral line radiation and its center-to-limb variation.
\end{abstract}

Keywords. Polarization, radiative transfer, scattering

\section{Introduction}

The radiation of some spectral lines emerging from the stellar atmospheres can be linearly polarized. This is due to resonance scattering of the anisotropic radiation in the line formation region. In addition, this scattering polarization can be modified by magnetic fields via the Hanle effect (Landi Degl'Innocenti \& Landolfi, 2004). This fact can be used for detection of inclined magnetic fields in the solar and stellar atmospheres.

However, the 1D plane-parallel geometry is a serious idealization of the real atmospheres that are not horizontally homogeneous (i.e., there are fluctuations of density, temperature and other plasma properties). Such fluctuations may modify the emergent line polarization in both high and low spatial resolution observations (the so-called symmetry breaking effect).

Neglecting symmetry breaking effects in modeling of radiation from stellar atmospheres could lead to misinterpretation of observations when compared with synthetic spectra. Moreover, studying radiative transfer under such conditions gives us valuable insight into the physics of formation of spectral line radiation in models, which are closer to the real stellar atmospheres.

Manso Sainz \& Trujillo Bueno (2011) studied a 2D problem of small horizontal inhomogeneities of the line opacity and source function. We study a more general case of inhomogeneities with arbitrarily large amplitudes. Such investigation requires to solve the full 3D non-LTE problem. To this end, we use the radiative transfer code PORTA of Štěpán \& Trujillo Bueno (2013). 


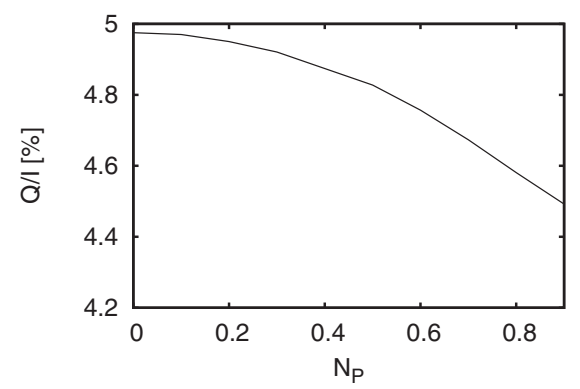

Figure 1. Spatially and azimuthally averaged $Q / I$ line-center amplitude as a function of $N_{P}$ for inclination $\mu=0.1$.

\section{Formulation of the problem}

For the purpose of this study, we choose an academic spectral line at $\lambda_{0}=5000 \AA$ of a two-level atom, with the angular momenta of the lower and upper levels being $J_{l}=0$ and $J_{u}=1$, respectively. The Einstein rate of spontaneous deexcitation is $A_{u l}=10^{8} \mathrm{~s}^{-1}$. A constant collisional destruction probability $\epsilon=10^{-4}$ is used.

As a reference model we use a semi-infinite isothermal $\left(T_{0}=6000 \mathrm{~K}\right)$ exponentially stratified atmosphere. The $3 \mathrm{D}$ model grid with $100 \times 100 \times 120$ points along the $x, y$, and $z$ axes, respectively, is a box with the dimensions of $D_{x} \times D_{y} \times D_{z}=1000 \times 1000 \times 2000 \mathrm{~km}^{3}$ with periodic boundary conditions in the horizontal directions. We simulate the horizontal inhomogeneities of the atmosphere by introducing sinusoidal fluctuations of the atomic density $N(x, y, z)$ :

$$
N(x, y, z)=\overline{N(z)}\left[1+N_{P} \sin \left(2 \pi \frac{x}{D_{x}}\right) \sin \left(2 \pi \frac{y}{D_{y}}\right)\right],
$$

where $D_{x}$ and $D_{y}$ are the periods of the density perturbation in the $x$ - and $y$-directions, respectively, and

$$
\overline{N(z)}=N_{0} \exp \left(-\frac{z-z_{0}}{\alpha}\right)
$$

is the mean atomic density at the height $z$. The dimensionless parameter $N_{P} \in[0,1]$ quantifies the amplitude of the perturbation: the case $N_{P}=0$ corresponds to the 1D iso-density atmosphere while in the case $N_{P}=1$, the perturbation is maximum. For the stratification parameter we choose $\alpha=75 \mathrm{~km}$.

For the temperature perturbations we use an analogous formula:

$$
T(x, y, z)=T_{0}\left[1+T_{P} \sin \left(2 \pi \frac{x}{x_{\max }}\right) \sin \left(2 \pi \frac{y}{y_{\max }}\right)\right] .
$$

In the present study, we always assume either the density or the temperature perturbation.

\section{Results}

Figure 1 shows the spatially and azimuthally integrated line-center $Q / I$ signal as a function of the density perturbation $N_{P}$ for the near-limb observation at $\mu=0.1$, where $\mu=\cos \theta$ with $\theta$ being the heliocentric angle. The linear polarization signal decreases with increasing amplitude of the perturbation.

The left panel of Figure 2 shows the center-to-limb variation of $Q / I$ for different values of $N_{P}$. The signal has also been averaged over all surface points and over all possible 

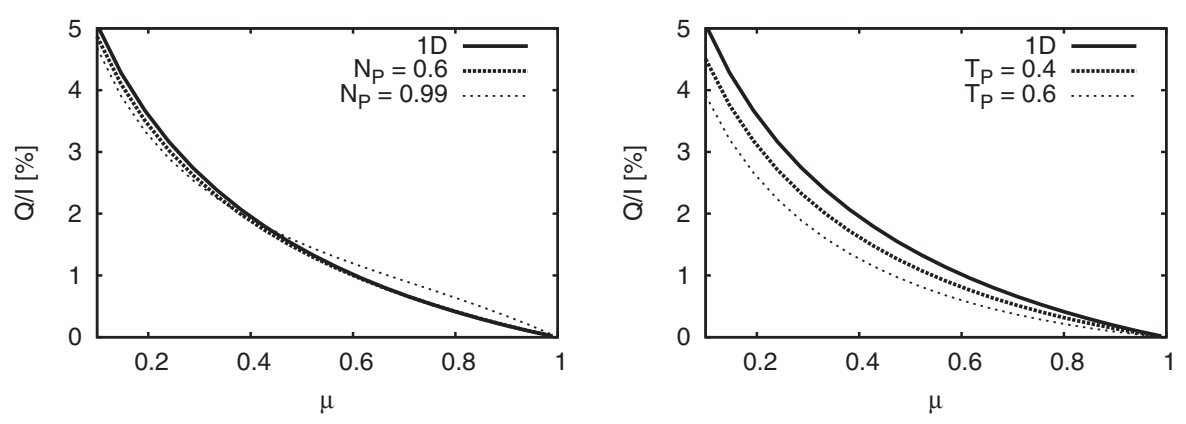

Figure 2. Center-to-limb variation of the azimuthally averaged $Q / I$ for various perturbation amplitudes. Left panel: density perturbations, right panel: temperature perturbations.
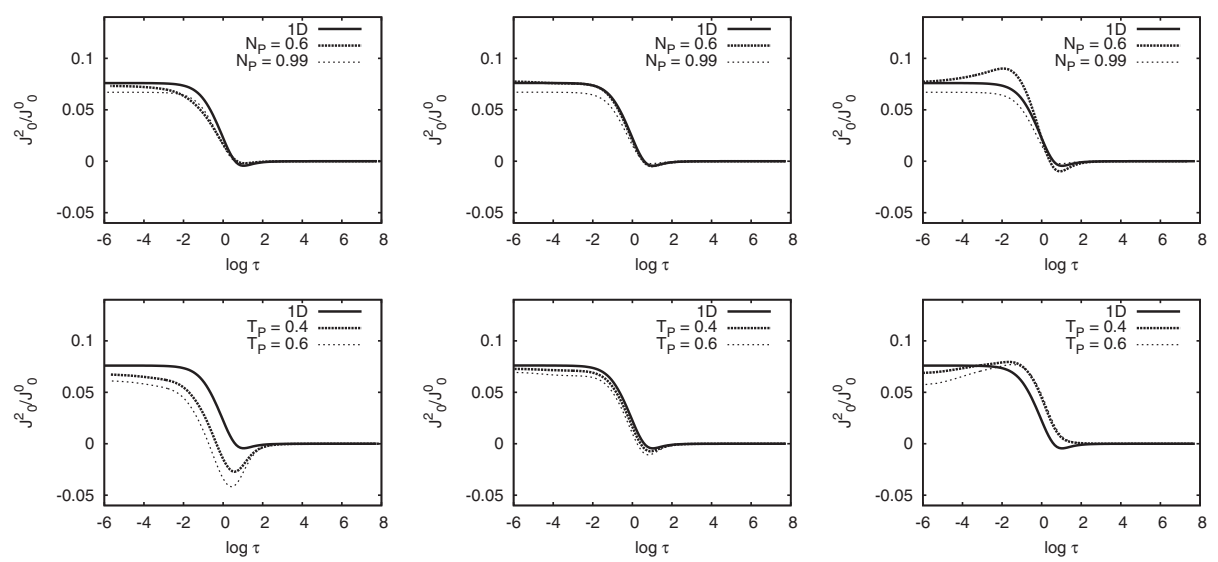

Figure 3. Radiation anisotropy factor $J_{0}^{2} / J_{0}^{0}$ as a function of atmospheric height (see text for more details). Top panels: density perturbations, bottom panels: temperature perturbations. Left panels: radiation anisotropy at the column of maximum perturbation (approximate coordinates $x=0.25 \mathrm{Mm}, y=0.25 \mathrm{Mm}$ ), middle panels: zero perturbation (approximate coordinates $x=0.5$ $\mathrm{Mm}, y=0.5 \mathrm{Mm}$ ), right panels: maximum negative perturbation (approximate coordinates $x=0.75 \mathrm{Mm}, y=0.25 \mathrm{Mm})$.

azimuths of the line of sight. In the $1 \mathrm{D}$ solution, the radiation anisotropy at the unit optical depth $\tau=1$ corresponding to the line of sight with $\mu=1$ is very low. However, as we increase the amplitude $N_{P}$, the average radiation field anisotropy decreases (see the top panels of Fig. 3). Interestingly, at larger $\mu$ values, the amplitude of the average $Q / I$ tends to be slightly larger in the perturbed model than in the $1 \mathrm{D}$ solution. This can be qualitatively explained by the fact that near the stellar disk center the $Q / I$ signal of the $1 \mathrm{D}$ model is very low and significant changes of the height of formation lead to deformation of the $\tau=1$ surface and, consequently, to creation of some additional polarization signal.

Similarly, the right panel of Figure 2 shows the the center-to-limb variation of $Q / I$ for different values of $T_{P}$.

The radiation anisotropy factor $J_{0}^{2} / J_{0}^{0}$ of the line as a function of optical depth is shown in Figure 3. For each amplitude of the perturbation shown, the fractional anisotropy $J_{0}^{2} / J_{0}^{0}$ has been plotted for the column in which the relative perturbation of the atomic density is $N_{P}$, zero and $-N_{P}$. Note that even for the columns where the density departures from the plane-parallel atmosphere are zero (i.e., columns for which one of the sine 

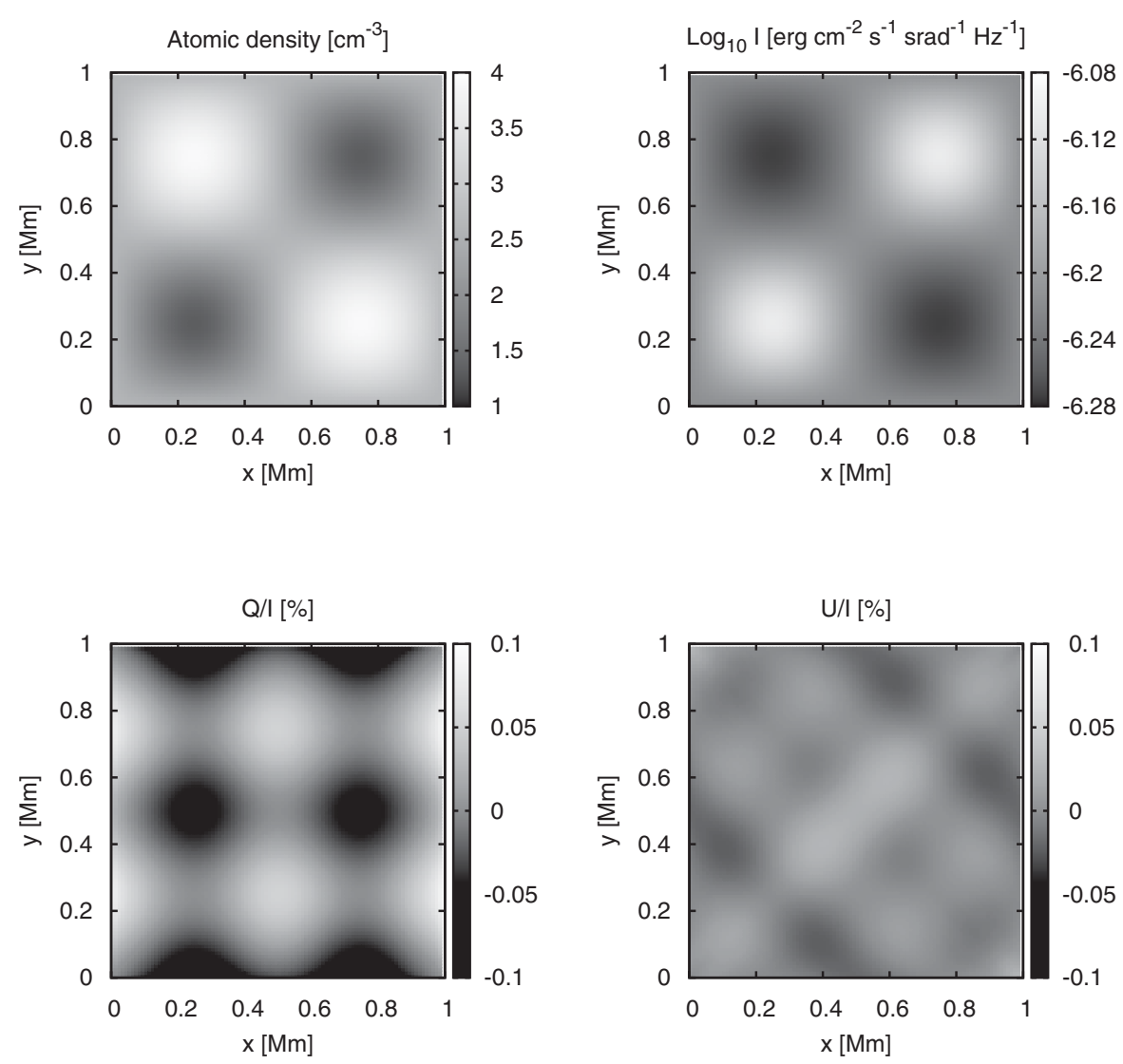

Figure 4. Quantities in the horizontal plane of the atmosphere with $N_{P}=0.6$ in the center of the line. Top left panel: map of variation of the atomic volume density in $\mathrm{cm}^{-3}$ near the surface of the atmosphere. Top right panel: emergent line intensity in the disk-center observation. Bottom panels: fractional linear polarization $Q / I$ (left) and $U / I$ (right).

functions in Eqs. (2.1) and (2.3) is identically zero) there is a difference in anisotropy with respect to the $1 \mathrm{D}$ solution.

Figures 4-6 show in greater detail the structure of the atmosphere and the properties of the emergent radiation for various amplitudes of the density and temperature perturbations.

Assuming perturbations according to Eqs. (2.1) and (2.3) we create density structure as shown in the top left panel of Figure 4, which corresponds to the line-intensity shown in the top right panel of the same figure. In both $Q / I$ and $U / I$ signals there are several points with a near-zero polarization due to approximate cylindrical symmetry of the radiation in these particular columns (the points of maximum and minimum perturbation) whereas maximal positive and negative polarizations are found in points with the largest gradients of physical properties of the model. The fact that the average $|U / I|$ amplitude is lower than average $|Q / I|$ amplitude is a consequence of the particular choice of the sinusoidal perturbations along the $x$ and $y$ axes that gives the model particular symmetry properties.

For illustration, Figure 5 shows vertical slices through the atmosphere. Perturbations are still quite small, considering that for $N_{P}=0.9$ the range of densities at a given height is of the order of one magnitude. However, in the more realistic models based on MHD 

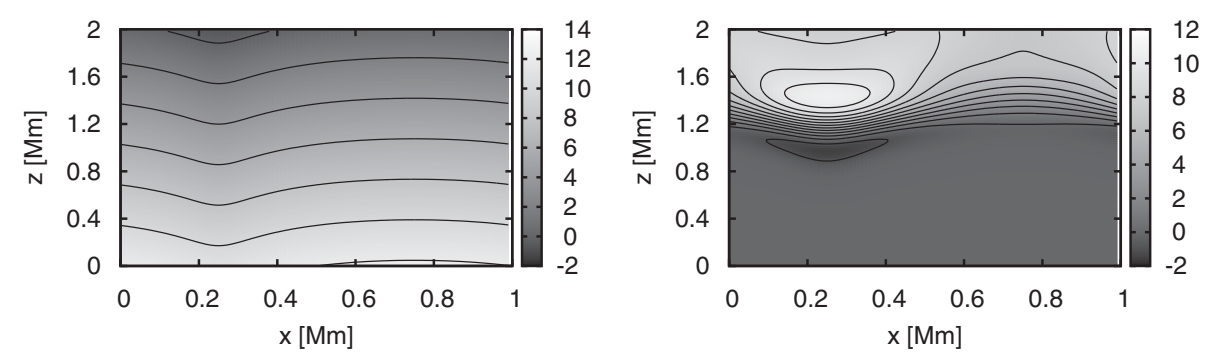

Figure 5. Physical quantities in a vertical $2 \mathrm{D}$ slice of the $3 \mathrm{D}$ atmosphere for $N_{P}=0.9$ at $y=D_{y} / 4$. Left panel: logarithm of the atomic density in cgs units. Right panel: radiation anisotropy factor $J_{0}^{2} / J_{0}^{0}$ [\%]. Solid lines refer to isolines of the corresponding quantities.
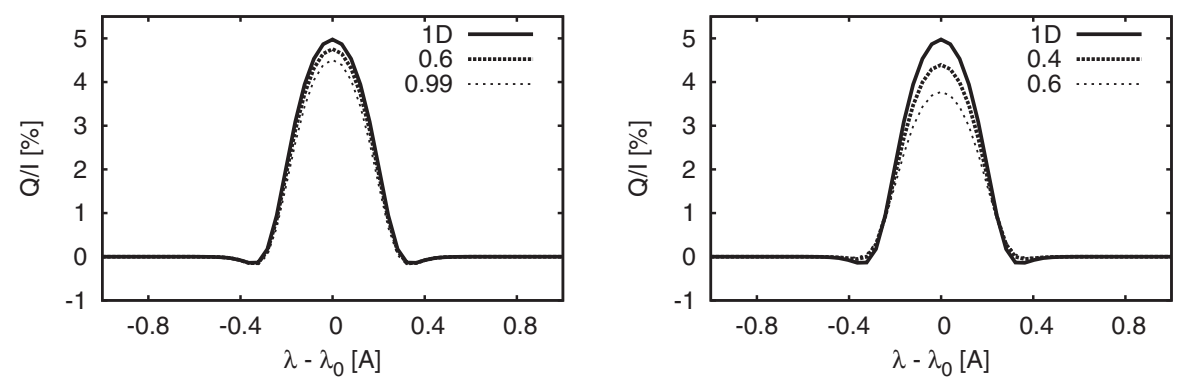

Figure 6. Fractional polarization $Q / I$ at $\mu=0.1$ (close to limb); labels indicate perturbation amplitudes. Left panel: density perturbations. Right panel: temperature perturbations.

simulations, the gradient on similar spatial scales could reach several more magnitudes (see, e.g., Štěpán 2015, this volume, Štěpán et al. 2012).

Spatially and azimuthally-averaged spectra are shown in Figure 6 . In the case of density perturbations the intensity itself is almost not influenced by the perturbation amplitude. However, in the center of the line the $Q / I$ signal differ by up to about 0.5 percent in contrast to the $1 \mathrm{D}$ solution. The temperature seem to have even larger impact for realistic perturbation amplitudes.

\section{Conclusions}

Our calculations show that horizontal perturbations of thermodynamic properties in stellar atmospheres may significantly influence the scattering polarization of the emergent radiation. In the simple models we have considered, the intensity is only slightly modified but the fractional linear polarization can be changed by up to a few percent. For both density and temperature perturbations, the fractional line polarization is decreased and the larger depolarizing effect seem to be due to the temperature perturbations. It is important to note that the presence of horizontal inhomogeneties not only affects the polarization pattern of the spatially averaged observations but it can significantly alter the spatially integrated spectra. Neglecting the role of horizontal inhomogeneities, such a change of linear polarization could be incorrectly interpreted as being due to the action of the Hanle effect and, consequently, it can lead to erroneous determination of the atmospheric magnetic fields.

It is important to note that horizontal perturbations of temperature and density in real stellar atmospheres are significantly more abrupt than in the simple models we have shown (see, e.g., Štěpán et al. 2012; Anusha \& Nagendra 2014). Accounting for 
multi-dimensional radiative transfer is therefore inevitable for many lines formed under the non-LTE conditions.

\section{Acknowledgements}

Financial support by the Grant Agency of the Czech Republic through grants 14-02385S and P209/12/P741, and the project RVO:67985815, as well as by the Spanish Ministry of Economy and Competitiveness through projects AYA2010-18029 (Solar Magnetism and Astrophysical Spectropolarimetry) and CONSOLIDER INGENIO CSD2009-00038 (Molecular Astrophysics: The Herschel and Alma Era) is gratefully acknowledged. Access to computing and storage facilities owned by parties and projects contributing to the National Grid Infrastructure MetaCentrum, provided under the programme "Projects of Large Infrastructure for Research, Development, and Innovations" (LM2010005), is greatly appreciated.

\section{References}

Anusha, L. S. \& Nagendra, K. N. 2014, in: K. N. Nagendra, J. O. Stenflo, Z. Q. Qu, \& M. Sampoorna (eds.), Solar Polarization 7, ASP Conf. Ser. 489 (San Francisco: ASP), p. 225 Landi Degl'Innocenti, E. \& Landolfi, M. 2004, Polarization in spectral lines, Kluwer, Dordrecht Manso Sainz, R. \& Trujillo Bueno, J. 2011, ApJ 743, 22

Štěpán, J., Trujillo Bueno, J., Carlsson, M., \& Leenaarts, J. 2012, Ap. Lett. 758, L43

Štěpán, J. \& Trujillo Bueno, J. 2013, A\&A 557, A143

Štěpán, J. 2015, this volume 